\title{
Local Averaging for Fast Handoffs in Cellular Networks
}

\author{
Brian L. Mark, Member, IEEE and Alexe E. Leu, Member, IEEE
}

\begin{abstract}
We propose a local averaging technique for processing the received pilot signal strength, which can significantly improve handoff performance in cellular networks. In handoff algorithms, the received pilot signal strength is typically averaged to diminish the undesirable effect of the fast fading component. Unfortunately, the averaging process can substantially alter the characteristics of path loss and shadowing components, causing increased handoff delay. The proposed local averaging method provides significant improvement for handoff delay performance, especially in the non-line-of-sight case, when the mobile station turns around a corner. An important feature of local averaging is that the handoff performance is insensitive to the speed of the mobile station, such that velocity estimation is not needed. We develop efficient numerical procedures to compute the handoff performance metrics under local averaging.
\end{abstract}

Index Terms-Cellular systems, radio propagation, communication system performance, handoff algorithms, smoothing methods, velocity measurement.

\section{INTRODUCTION}

$\mathbf{I}$ $\mathrm{N}$ cellular networks, the handoff algorithm determines the connectivity of a mobile station (MS) to an active set of base stations. In this paper, we focus on the handoff process at the physical layer, in which handoff decisions are based on measurements of pilot signal strengths received from candidate base stations. The pilot signal strength is modeled by three components: path loss, shadowing, and fast fading. The signal measurements are typically processed using averaging filters to reduce the fast fading fluctuations and hence, the number of unnecessary handoffs experienced as the MS moves in the network.

Ideally, the processed signal strength should closely track the contribution of the two propagation attenuation components, i.e., path loss and shadowing. Unfortunately, conventional averaging methods tend to substantially alter the path loss and shadowing statistics encountered in the received signal, resulting in large handoff delays. Under conventional averaging, handoff decisions are made at the sampling instants such that each handoff decision is based on an average of the

Manuscript received February 4, 2004; revised July 8, 2006; accepted October 28, 2006. The editor coordinating the review of this paper and approving it for publication is L. Wang. This work was supported in part by the U.S. National Science Foundation under Grant 0133390 and by a grant from Northrop Grumman Mission Systems. An early version of this work was presented in part at the 10th ACM/IEEE Int. Symp. on Modeling, Analysis and Simulation of Computer and Telecommunications Systems (MASCOTS), Oct. 11-16, 2002.

B. L. Mark is with the Department of Electrical and Computer Engineering, The Volgenau School of Information Technology \& Engineering, George Mason University, Fairfax, VA 22030 (e-mail: bmark@gmu.edu).

A. E. Leu is with Shared Spectrum Company, Vienna, VA 22182. (e-mail: aleu@sharedspectrum.com).

Digital Object Identifier 10.1109/TWC.2007.04080.

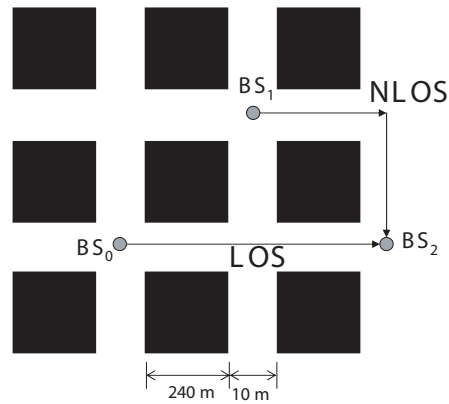

Fig. 1. Line-Of-Sight (LOS) and Non-LOS (NLOS) trajectories in a microcellular network.

signal measurements at the current and past handoff instants. When the MS travels at higher speeds, the distance between handoff instants increases proportionately and consequently, the averaged signal measurements do not track the actual path loss and shadowing components of the pilot signal.

This problem becomes especially acute when the mobile station leaves the line-of-sight (LOS) of a base station to which it is assigned. Fig. 1 shows a LOS trajectory from base station $B S_{0}$ to $B S_{2}$ and a non-LOS (NLOS) trajectory from $B S_{1}$ to $B S_{2}$. Corner effects, which have been verified by measurements [1], are characterized by a $20-30 \mathrm{~dB}$ drop of signal level in 10-20 meters and appear when the MS turns around a corner and loses the LOS path to the BS. In corner effect scenarios, the handoff decision is based on an overly deteriorated version of the pilot signal strength. To avoid deterioration of the pilot signal strength, the window size used in conventional averaging schemes should ideally be a function of the velocity of the MS. Velocity adaptive handoff algorithms [2], [3] adjust the window size dynamically based on the estimated speed of the mobile station. However, such algorithms require accurate velocity estimation, which may not always be feasible in practice (cf. [4]).

In this paper, we propose a local averaging technique for processing the received signal strength measurements used to make handoff decisions. Local averaging has the desirable feature of being relatively insensitive to the speed of the MS, obviating the need for accurate velocity estimation or adaptive adjustment of the averaging window length. In local averaging, the received signal is sampled at a faster rate than the rate at which handoff decisions are made. That is, the sampling time interval is chosen to be smaller than the handoff decision interval, as opposed to conventional averaging in which the signal is sampled at the handoff decision instants. Handoff decisions are based on a local average of the signal samples 


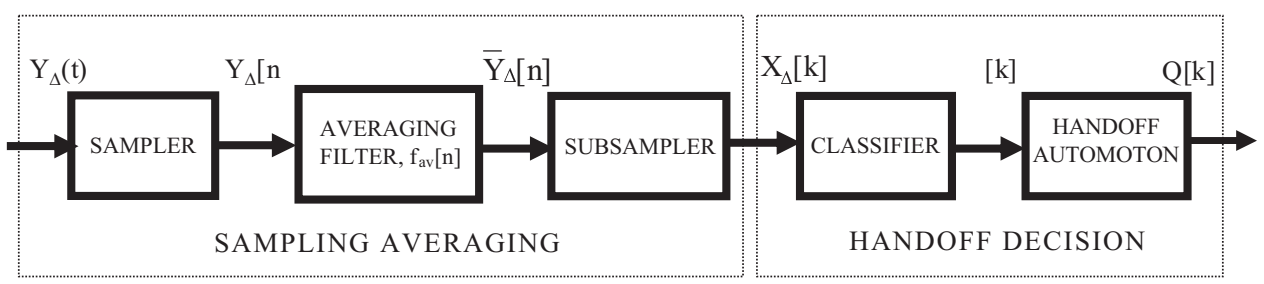

Fig. 2. Framework for handoff analysis.

occurring between successive handoff decision epochs. Local averaging reduces the fast fading fluctuations of the received signal strength without substantially altering the path loss and shadowing components of the signal.

By examining the properties of the locally averaged signal strength, we show why local averaging yields superior handoff performance. Further, we develop an efficient numerical procedure for evaluating handoff performance under local averaging. Our numerical results confirm that local averaging significantly improves handoff performance with respect to conventional averaging methods. Moreover, handoff performance under local averaging is relatively insensitive to the speed of the MS such that there is no need for velocity estimation.

Approximate methods for evaluating the performance of hysteresis-based handoff algorithms using conventional averaging techniques were developed in [2], [5], [6]. These studies of handoff performance showed that the tradeoff between number of handoffs and handoff delay depends strongly on the size of the averaging window. The choice of window size also depends on the sampling interval. In [7], a discretetime handoff analysis approach was developed that provided new insight into the effect of averaging on hard handoff performance. In particular, averaging distorts the path loss and shadowing components of the signal, which increases the handoff delay. This observation motivated the present study on local averaging.

A key assumption made in a number of handoff algorithms proposed in the literature [8], [9], is that the fast fading component can be removed from the sample pilot signal strength measurement through low pass filtering without affecting the path loss and shadowing components of the signal propagation model. However, the impact of low pass filtering (i.e., averaging) is usually not taken into account. Our proposed local averaging technique in effect reduces the fast fading fluctuations without appreciably modifying the path loss and shadowing components. Thus, local averaging can be applied to improve the performance of any handoff algorithm that uses sampled pilot strength measurements. In the present paper, we focus on the class of hysteresis-based handoff algorithms due to their simple implementation in practice and analytical tractability.

The corner effect was studied in [3], where it was concluded that a short averaging window and a large hysteresis level should be used to accommodate rapid changes in the mean signal strength and to avoid unnecessary handoffs. The handoff algorithms proposed in [3] adapt the averaging window length to the speed of the mobile station. The pattern recognitionbased algorithm in [10] improves handoff performance in the presence of corner effects, but it too relies on the assumption that the velocity of the mobile station can be estimated accurately so that the averaging window can be adjusted proportionately. In [2], an adaptive averaging methodology for handoff is developed, which also relies on velocity estimation. The local averaging technique proposed in the present paper significantly improves handoff performance in corner effect scenarios without relying on knowledge of the mobile velocity.

The remainder of the paper is organized as follows. Section II describes a discrete-time framework for handoff analysis, which provides the basis for evaluating the impact of averaging on handoff performance. Section III introduces the local averaging technique and gives an important result concerning the statistics of the locally averaged signal strength. Section IV develops a recursive procedure for calculating the handoff and cell assignment probabilities under local averaging. Numerical results showing the performance gains achievable with local averaging are presented in Section V. Finally, the paper is concluded in Section VI.

\section{A FRAMEWORK FOR HANDOFF ANALYSIS}

In this section, we describe the underlying signal propagation model assumed in the paper and present a conceptual framework (cf. [7], [11]) for analyzing the handoff algorithm in terms of the five components illustrated in Fig. 2: sampler, averaging filter, subsampler, classifier, and handoff automaton. The first three components perform the task of sampling and averaging the pilot signal. The last two components comprise the actual handoff decision algorithm, which takes the processed pilot signal strength samples as inputs and produces a decision at each handoff decision epoch. The handoff framework provides a basis for making performance comparisons among different handoff algorithms with respect to the averaging technique used. In particular, we explain the concept of local averaging versus conventional averaging in the context of this framework.

\section{A. Propagation Model}

The cellular network consists of a set of base stations. The $i$ th base station, $B S_{i}$, located by a position vector $\mathbf{b}_{i}$, lies at the center of its associated cell $i$. The coverage area of cell $i$ is determined by the pilot signal strength received from $B S_{i}$. The received signal strength from the $i$ th base station is modeled in $\mathrm{dB}$ representation as follows [12]:

$$
Y_{i}(t)=\Gamma_{i}(t)+W_{i}(t)+R_{i}(t),
$$

where $\Gamma_{i}(t), W_{i}(t)$, and $R_{i}(t)$ represent, respectively, the path loss, lognormal shadowing, and fast fading components. 
For LOS propagation we assume a two-slope path loss model [13], given by

$$
\begin{aligned}
\Gamma_{L O S, i}(t) & =\nu_{i}-10 \mu_{i} \log _{10}\left(\left\|\mathbf{r}(t)-\mathbf{b}_{i}\right\|\right) \\
& -10 \beta_{i} \log _{10}\left(1+\frac{\left\|\mathbf{r}(t)-\mathbf{b}_{i}\right\|}{g}\right),
\end{aligned}
$$

where $\nu_{i}$ is the base station transmit power, $\mu_{i}$ and $\beta_{i}$ determine the path loss exponent, and $g$ is called the breakpoint. Typical parameter values for an urban environment are: $\mu_{i}=$ $2, \beta_{i}=1$ or 2 , and $150 \mathrm{~m} \leq g \leq 500 \mathrm{~m}$. For NLOS propagation, we assume the following model proposed by Grimlund [1]:

$$
\begin{aligned}
\Gamma_{N L O S, i}(t) & =\Gamma_{L O S, i}(t) u\left(-v t+D_{c}\right)+\left[\Gamma_{L O S, i}\left(D_{c} / v\right)\right. \\
& \left.+\Gamma_{L O S, i}\left(t-D_{c} / v\right)\right] u\left(v t-D_{c}\right)
\end{aligned}
$$

where $u(t)$ is the unit step function, $v$ is the mobile speed, and $D_{c}$ is the distance from the base station to the corner of a street block, e.g., in the NLOS trajectory shown in Fig. $1, D_{c}=$ $255 \mathrm{~m}$. In the NLOS model given in (3), LOS propagation is assumed until the mobile rounds the corner of an obstacle, after which LOS propagation is assumed from an imaginary transmitter located at the corner having power equal to that received from the serving base station at the corner.

The shadowing component, $W_{i}(t)$, is a Gaussian process with an exponentially decaying autocorrelation function [14]:

$$
R_{W_{i}}(\tau)=\sigma_{W_{i}}^{2} \exp \left(-\frac{v \tau}{d_{0}}\right)
$$

where $\sigma_{W_{i}}$ is the standard deviation of the shadowing signal strength, $v$ is the mobile speed, and the constant $d_{0}$ is called the decay factor. The fast fading component, $R_{i}(t)$, is the logarithmic scale representation for the envelope of a complex process $z_{i}(t)=z_{i, I}(t)+j z_{i, Q}(t)$ :

$$
R_{i}(t)=10 \log _{10} \sqrt{\left|z_{i, I}(t)\right|^{2}+\left|z_{i, Q}(t)\right|^{2}},
$$

where the in-phase and quadrature components $z_{i, I}(t)$ and $z_{i, Q}(t)$ are independent Gaussian random processes. The two components, $z_{i, I}(t)$ and $z_{i, Q}(t)$, are independent when the angle of incidence of the arriving plane waves is uniformly distributed over $[-\pi, \pi]$. This model is commonly referred to as Clarke's two-dimensional isotropic scattering model [15]. In the NLOS scenario, $z_{i, I}(t)$ and $z_{i, Q}(t)$ are independent, identically distributed zero-mean Gaussian random processes and the magnitude of the received complex envelope has a Rayleigh distribution at any time $t$. In the LOS case, $z_{i, I}(t)$ and $z_{i, Q}(t)$ are independent Gaussian random processes with non-zero means and the magnitude of the received complex envelope has a Ricean distribution. As will be discussed in Section III-B, the proposed local averaging technique reduces the fast fading component in both NLOS and LOS scenarios.

\section{B. Sampler}

The received pilot signals are sampled at discrete time instants $t_{n}=n \tau_{s}$ where $n$ is a nonnegative integer and $\tau_{s}$ is the sampling interval. The sampling distance is defined by $d_{s}=v \tau_{s}$, where $v$ is the mobile speed. In the discrete-time representation, the received signal strength from the $i$ th base station at time $t_{n}$ is given by

$$
Y_{i}[n]=\Gamma_{i}[n]+W_{i}[n]+R_{i}[n],
$$

where $Y_{i}[n] \triangleq Y_{i}\left(t_{n}\right), \Gamma_{i}[n] \triangleq \Gamma_{i}\left(t_{n}\right), W_{i}[n] \triangleq W_{i}\left(t_{n}\right)$, and $R_{i}[n] \triangleq R_{i}\left(t_{n}\right)$. When the sampling interval satisfies

$$
\tau_{s} \geq 0.38 \lambda_{c} / v,
$$

where $\lambda_{c}$ is the carrier wavelength, the fast fading samples $\left\{R_{i}[n]\right\}$ can be treated as independent, identically distributed Rayleigh or Ricean random variables (cf. [15]). We shall assume throughout the paper that the sampling interval satisfies (7).

\section{Averaging Filter}

The purpose of the averaging filter is to reduce the fast fading component $R_{i}[n]$ in the received signal strength $Y_{i}[n]$. Let $\left\{f_{a v}[n]\right\}_{n \geq 0}$ denote the discrete-time averaging window. We assume that $\sum_{n=0}^{\infty} f_{a v}[n]=1$. The averaged pilot signal strength of $B S_{i}$ at time $n$ is denoted by

$$
\bar{Y}_{i}[n] \triangleq f_{a v}[n] \star Y_{i}[n]=\bar{\Gamma}_{i}[n]+\bar{W}_{i}[n]+\bar{R}_{i}[n],
$$

where $\star$ denotes discrete-time convolution and the averaged versions of the path loss, shadowing, and fast fading components are denoted by $\bar{\Gamma}_{i}[n], \bar{W}_{i}[n]$, and $\bar{R}_{i}[n]$, respectively.

\section{Subsampler}

The time interval between handoff decisions is denoted by $\tau_{h}$. In practice, the handoff interval cannot be chosen to be too small since the bandwidth for signaling is limited. For example, in the GSM standard, $\tau_{h}=0.48 \mathrm{~s}$. We shall assume that $\tau_{h}$ is an integer multiple of the sampling interval $\tau_{s}$, i.e., $\tau_{h}=q \tau_{s}$ where $q \geq 1$. Handoff decisions are based on the value of the averaged pilot signal strength $\bar{Y}_{i}[n]$ at the handoff decision instants $n=l q$, where $l \geq 0$ is an integer. To analyze handoff performance, it is convenient to consider the subsampled sequence

$$
X_{i}[k] \triangleq \bar{Y}[k q]=\mu_{i}[k]+S_{i}[k]+Z_{i}[k],
$$

where $\mu_{i}[k] \triangleq \bar{\Gamma}_{i}[k q], S_{i}[k] \triangleq \bar{W}_{i}[k q]$, and $Z_{i}[k] \triangleq \bar{R}_{i}[k q]$ are the subsampled versions of the path loss, shadowing, and fast fading components after averaging. In conventional handoff algorithms, the sampling interval and handoff decision interval are the same, i.e., $q=1$, and there is no need to subsample the signal $\bar{Y}_{i}[n]$.

We now define the concept of local averaging in contrast to the conventional method of averaging the pilot signal strength in handoff algorithms. Local averaging is defined by the following two properties:

1) The sampling interval is less than the handoff interval, i.e., $q>1$.

2) The averaging filter defined by $f_{a v}[n]$ satisfies $f_{a v}[n]=$ 0 for $n \geq q$. Equivalently, the averaging filter has finite support of length $N_{a v}<q$.

In other words, the signal value $X_{i}[k]$ used in making the $k$ th handoff decision is a local average of the sampled pilot signal 
strength values $Y_{i}[n]$ in the range $(k-1) q<n \leq k q$. By contrast, in conventional averaging, the signal value $X_{i}[k]$ is an average of the pilot signal strength values taken at the handoff decision instants. We shall show that handoff performance can be improved significantly by replacing conventional averaging with local averaging.

\section{E. Signal Classifier}

The role of the signal classifier is to map the processed pilot signal, $X_{i}[k]$, into a sequence of symbols. The definition of the signal classifier depends on the particular handoff algorithm. In the remainder of the paper, we shall focus on the class of hysteresis-based hard handoff algorithms (cf. [5]). In this type of handoff algorithm, the mobile station (MS) selects one of two candidate base stations, $B S_{i}$ and $B S_{j}$, based on comparing the relative signal strength $X_{\Delta}[k]=X_{i}[k]-X_{j}[k]$ to hysteresis levels $h_{i}$ and $-h_{j}$. If the MS is assigned to $B S_{i}$ at time $k-1$, a handoff to $B S_{j}$ occurs at time $k$ if $X_{\Delta}[k] \leq-h_{j}$. Conversely, if the MS is assigned to $B S_{j}$ at time $k-1$, a handoff from $B S_{j}$ to $B S_{i}$ occurs at time $k$ if $X_{\Delta}[k] \geq h_{i}$.

The hysteresis levels partition the real line into three disjoint intervals defined as follows:

$$
\mathcal{I}=\left[h_{i}, \infty\right), \quad \mathcal{H}=\left(-h_{j}, h_{i}\right), \quad \mathcal{J}=\left(-\infty,-h_{j}\right],
$$

where $\mathcal{I}$ is called the assignment region for $B S_{i}, \mathcal{H}$ is the hysteresis region, and $\mathcal{J}$ is the assignment region for $B S_{j}$. The handoff behavior of the MS can be couched in terms of a classification of the value $X_{\Delta}[k]$ into one of these three intervals. The assignment regions at system initialization time $t_{0}$ are $\mathcal{I}_{0}=[0, \infty)$ and $\mathcal{J}_{0}=(-\infty, 0)$.

The signal classifier maps the received signal $X_{\Delta}[k]$ into a symbol $L[k]$ for $k \geq 1$ defined on the alphabet $\mathcal{S}=\{I, H, J\}$ as follows:

$$
L[k]= \begin{cases}I, & \text { if } X_{\Delta}[k] \in \mathcal{I} \\ H, & \text { if } X_{\Delta}[k] \in \mathcal{H} \\ J, & \text { if } X_{\Delta}[k] \in \mathcal{J}\end{cases}
$$

for $k \geq 1$ and

$$
L[0]= \begin{cases}I_{0}, & \text { if } X_{\Delta}[k] \in \mathcal{I}_{0}, \\ J_{0}, & \text { if } X_{\Delta}[k] \in \mathcal{J}_{0} .\end{cases}
$$

We shall treat subsequences of the sequence $\{L[k]\}_{k \geq 0}$ as strings over the alphabet $\mathcal{S}$. For $L \in \mathcal{S}$, we denote by $L^{k}$ the string $L \ldots L$ ( $k$ times), when $k \geq 1$ and $L^{0}$ denotes the null string.

\section{F. Handoff Automaton}

The handoff mechanism can be specified by a finite automaton $\mathcal{A}=(Q, \Sigma, \nu)$, where:

- $Q=\left\{q_{i}, q_{j}\right\}$, where state $q_{i}$ represents assignment of the MS to $B S_{i}$ and state $q_{j}$ represents assignment to $B S_{j}$.

- $\Sigma=\{I, H, J\}$ is the input alphabet.

- $\nu$ is the state transition function, $\nu: Q \times \Sigma \longrightarrow Q$, that assigns the next state given the current state and input symbol. The transition function for hysteresis-based hard handoff, $\nu(q, l)$, is defined as follows:

$$
\nu(q, l) \triangleq \begin{cases}q_{i}, & \text { if } l=I, \\ q, & \text { if } l=H, \\ q_{j}, & \text { if } l=J .\end{cases}
$$

\section{Properties of the Locally Averaged Signal}

The signal propagation model discussed in Section II-A is defined in terms of the path loss and statistical characterizations of the shadowing and fast fading components. Ideally, the handoff decision should be determined by the path loss and shadowing components of the received signal strength, since the fast fading fluctuations occur on a very short time-scale. Hence, the ideal averaging method for optimum handoff performance would eliminate the fast fading component without altering the path loss and shadowing components.

Conventional averaging methods can substantially modify the path loss and the statistical characteristics of the shadowing component, which may result in poor handoff performance especially when the mobile turns around corners. In such situations, the sudden changes in path loss and shadowing tend to be smoothed out too much by the averaging filter, making it difficult to track the true path loss encountered by the pilot signal. In this section, we show that local averaging essentially preserves the characteristics of path loss and shadowing components of the received pilot signal while still reducing the fast fading component to a sufficiently small level.

\section{A. Path Loss Component}

The concept of local averaging can be formalized in terms of local averages defined as follows:

$$
\left\langle\Gamma, u_{k}\right\rangle \triangleq \int \Gamma(t) u_{k}(t) d t
$$

where $\left\{u_{k}\right\}$ is a collection of nonnegative averaging functions, which satisfy

$$
\operatorname{supp} u_{k} \subset\left[t_{k}-\frac{\delta}{2}, t_{k}+\frac{\delta}{2}\right] \text { and } \int u_{k}(t) d t=1,
$$

where supp $u_{k}$ denotes the support of $u_{k}$. Intuitively, one should be able to obtain a good approximation of the original signal $\Gamma(t)$ from the local averages if $\delta$ is sufficiently small. Indeed, results have been obtained (cf. [16]) which provide sufficient conditions on $\delta$ and the sampling interval $\tau_{s}$ for $\Gamma_{i}(t)$ to be uniquely reconstructed from local averages. In particular, suppose that the averaging functions are even and nonincreasing on $\left[0, \frac{\delta}{2}\right]$, e.g.,

$$
u_{k}(t)=\frac{1}{\delta} 1_{\left[t_{k}-\frac{\delta}{2}, t_{k}+\frac{\delta}{2}\right]}(t),
$$

where $1_{A}$ denotes the indicator function on the set $A$. In [16] (Theorem 2.3), it is shown that a function $\Gamma(t)$, bandlimited to $[-\Omega, \Omega]$ can be uniquely reconstructed from the local averages $\left\langle\Gamma, u_{k}\right\rangle$ with $t_{k}=\frac{k \pi}{\Omega}$ if $0<\delta<\frac{1.8830453 \pi}{\Omega}$. This result can be viewed as a generalization of the classical Shannon sampling theorem.

The local averaging scheme described in Section II may be viewed as a special case in which the averaging functions are defined as follows:

$$
u_{k}\left(t+t_{k}\right)=\frac{1}{N_{a v}} \sum_{k=1}^{N_{a v}} \delta\left(t-k \tau_{s}-N_{a v} \tau_{s} / 2\right),
$$

where $\delta(t)$ denotes the Dirac delta function, $\tau_{s}$ is the subsampling interval, and $N_{a v}$ is the number of samples used for 


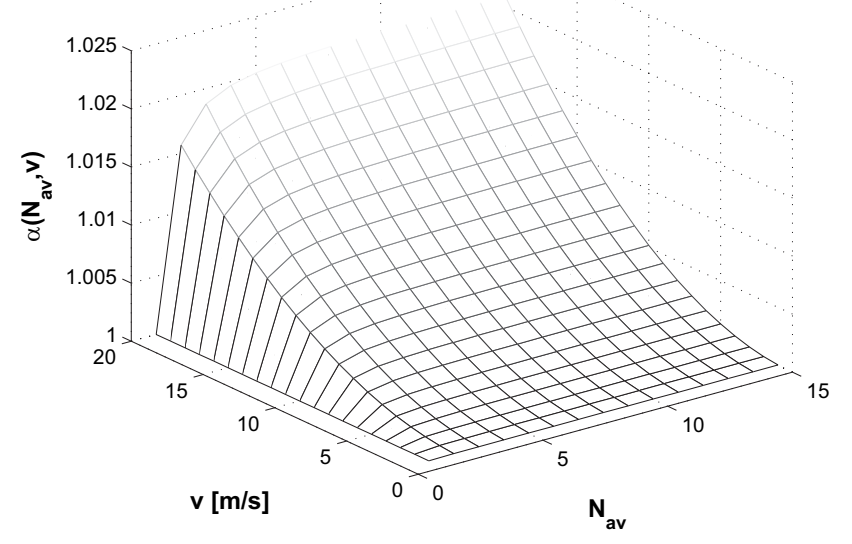

Fig. 3. The shadowing autocorrelation scaling factor $\alpha\left(N_{a v}, v\right)$ for a rectangular averaging filter.

local averaging. By appealing to the result of [16], one sees that the local averaging scheme essentially preserves the path loss component of the received signal strength measurements.

\section{B. Autocovariance Function}

Let $W_{\Delta}[n] \triangleq W_{i}[n]-W_{j}[n]$ denote the $n$th sample of the relative shadowing component prior to averaging. Let $S_{\Delta}[k] \triangleq$ $S_{i}[k]-S_{j}[k]$ and $Z_{\Delta}[k] \triangleq Z_{i}[k]-Z_{j}[k]$ denote the relative shadowing and fast fading component samples after averaging at the $k$ th handoff decision epoch. The following proposition shows that under local averaging, the autocovariance function $K_{X_{\Delta}}(k)$ of the process $\left\{X_{\Delta}[k]\right\}$ is essentially the same as the autocovariance function $K_{W_{\Delta}}(k)$ of the relative shadowing component, $\left\{W_{\Delta}[k q]\right\}$.

Proposition 1: Under local averaging with an averaging filter $f_{a v}[n]$,

$$
K_{X_{\Delta}}(l)=K_{S_{\Delta}}(l)+K_{Z_{\Delta}}(l),
$$

where $K_{S_{\Delta}}(l)=\alpha\left(N_{a v}, v\right) K_{W_{\Delta}}(l q)$ and $K_{Z_{\Delta}}(l)=$ $\beta\left(N_{a v}\right) \delta[l q]$ with

$$
\begin{aligned}
\alpha\left(N_{a v}, v\right) & =\sum_{j=0}^{N_{a v}-1} \sum_{l=-j}^{N_{a v}-1-j} f_{a v}[l+j] f_{a v}[j] e^{-l v \tau_{s} / d_{0}}, \\
\beta\left(N_{a v}\right) & =\frac{50}{3(\ln 10)^{2}} f_{a v}[0], \\
K_{W_{\Delta}}(l q) & =\sigma_{W_{\Delta}}^{2} e^{-l q v \tau_{s} / d_{0}},
\end{aligned}
$$

where $\sigma_{W_{\Delta}}^{2}=2 \sigma_{W_{i}}^{2}$ and $\delta[k]$ denotes the Kronecker delta function defined by $\delta[0]=1$ and $\delta[k]=0$ for $k \neq 0$. Here, $v$ denotes the MS speed, and $d_{0}$ is the decay factor associated with the autocorrelation function of the shadowing component.

Proposition 1 (see Appendix A for a proof) implies that the process $\left\{S_{\Delta}[k]\right\}$ can be expressed in terms of a first-order autoregressive (AR) model as follows:

$$
S_{\Delta}[k]=\tilde{a} S_{\Delta}[k-1]+U[k], \quad k \geq 0,
$$

where $\{U[k]\}$ is a zero mean, stationary white Gaussian process with variance $\sigma_{U}^{2}$ and

$$
\tilde{a}=\sqrt{\alpha\left(N_{a v}, v\right)} e^{-v \tau_{h} d_{o}}, \quad \sigma_{U}^{2}=\left(1-\tilde{a}^{2}\right) \sigma_{W_{\Delta}}^{2} .
$$

The AR representation (17) of $S_{\Delta}[k]$ greatly simplifies the handoff analysis as we shall see in the next section.

For concreteness, consider the case when the averaging filter is rectangular, i.e., defined by $f_{a v}[n]=1 / N_{a v}, \quad n=$ $0,1, \cdots, N_{a v}-1$. In this case,

$$
\alpha\left(N_{a v}, v\right)=\frac{1}{N_{a v}^{2}} \sum_{l=-N_{a v}+1}^{N_{a v}-1}\left(N_{a v}-|l|\right) e^{l v \tau_{s} / d_{0}} .
$$

Fig. 3 plots the scaling factor in (18) with $N_{a v}$ ranging from 1 to 15 . From the figure, we observe that the variation of the surface is at most $2 \%$ from minimum value of 1 , when the MS speed ranges from $1 \mathrm{~m} / \mathrm{s}$ to $20 \mathrm{~m} / \mathrm{s}$. Since $\alpha\left(N_{a v}, v\right) \approx 1$, we have that $K_{S_{\Delta}}(l) \approx K_{W_{\Delta}}(l q)$. Thus, the statistical properties of the shadowing component are essentially invariant under local averaging. For the rectangular averaging window, $\beta\left(N_{a v}\right)$ in (15), which determines the variance of the residual fast fading component, is inversely proportional to the parameter $N_{a v}$. Hence, local averaging can be parameterized so as to reduce the fast fading component substantially. We remark that this property holds for both the LOS and NLOS fast fading models discussed in Section II-A.

\section{HANDOFF ANALYSIS}

In this section, we develop numerical procedures to calculate the handoff performance metrics of interest when local averaging is employed.

\section{A. Classifier Output String}

The output of the signal classifier at each time $k$ is a symbol $L[k]$ from the alphabet $\mathcal{S}=\{I, H, J\}$, representing the assignment region. As the mobile moves along a trajectory, the classifier outputs a string of letters $\{L[k]: k=0, \ldots, K\}$. We use the following notation to denote the substring consisting of $L[k]$ and the preceding $r-1$ characters in the output string:

$$
L[k \backslash r] \triangleq(L[k-r+1], \ldots, L[k]) .
$$

Let $\mathcal{S}^{r}$ be the Cartesian product of the set $\mathcal{S}$ with itself, $r$ times. We denote the probability that the string $L[k \backslash r] \in \mathcal{S}^{r}$ occurs at the output of the signal classifier at time $k$ as follows:

$$
p_{k}(L[k \backslash r]) \triangleq P\left\{X_{\Delta}[k \backslash r] \in L[k \backslash r]\right\},
$$

where $X_{\Delta}[k \backslash r]=\left(X_{\Delta}[k-r+1], \ldots, X_{\Delta}[k]\right)$.

To evaluate probabilities of the form (20), we introduce a family of operators $\mathcal{F}=\left\{\mathbf{I}_{k}, \mathbf{H}_{k}, \mathbf{J}_{k}\right\}_{k \geq 0}$ defined on the space of probability density functions (pdfs) $\Psi: \mathcal{R} \rightarrow[0,1]$. The operators $\mathbf{I}_{0}, \mathbf{J}_{0}$, and $\mathbf{H}_{0}$ are associated with the assignment regions $\mathcal{I}_{0}, \mathcal{J}_{0}$, and $\mathcal{H}_{0} \triangleq \emptyset$, respectively. For $k \geq 1$, the operators $\mathbf{I}_{k}, \mathbf{J}_{k}$, and $\mathbf{H}_{k}$ are associated with the assignment regions $\mathcal{I}, \mathcal{J}$, and $\mathcal{H}$, respectively. The operator $\mathbf{L}_{k} \in \mathcal{F}$ is defined as follows (cf. [7]):

$$
\mathbf{L}_{k} \Psi(s)=\iint_{u+z \in L} \Psi(u) f_{k+1}(s \mid u) f_{Z_{k-1}}(z) d u d z,
$$


where $\mathcal{L}_{k}$ corresponds to the particular assignment region in the set $\left\{\mathcal{I}, \mathcal{H}, \mathcal{J}, \mathcal{I}_{0}, \mathcal{J}_{0}\right\}$ associated with $\mathbf{L}_{k}$. Here,

$$
f_{k}(s \mid u)=\frac{1}{\sigma_{U} \sqrt{2 \pi}} \exp \left\{-\frac{(s-\tau)^{2}}{2 \sigma_{U}^{2}}\right\}
$$

is the conditional density of $\mu_{\Delta}[k]+S_{\Delta}[k]$ given that $\mu_{\Delta}[k-$ $1]+S_{\Delta}[k-1]=u$, and $f_{Z_{k-1}}(z)$ denotes the pdf of the residual relative fast fading component, $Z_{\Delta}[k]$. Note that $f_{Z_{k}}(z)=f_{Z_{i, k}}(z) \star f_{Z_{j, k}}(-z)$, where $f_{Z_{i, k}}(z)$ and $f_{Z_{j, k}}(z)$ are the pdfs of $Z_{i}[k]$ and $Z_{j}[k]$, respectively. Hence, $f_{Z_{k}}(z)$ is an even function. Applying the central limit theorem, the pdf $f_{Z_{k}}(z)$ can be approximated by a Gaussian pdf with mean zero and variance $\frac{50 \pi^{2}}{3 N_{a v}(\ln 10)^{2}}$.

By using the fact that the pdf $f_{Z_{k}}(z)$ is an even function, one can show that the operator $\mathbf{H}_{k}$ can be simplified as follows:

Proposition 2:

$$
\mathbf{H}_{k} \Psi(s)=\int_{\mathcal{H}} \Psi(u) f_{k}(s \mid u) d u .
$$

Proposition 2 shows that the operator $\mathbf{H}_{k}$ is invariant with respect to the pdf, $f_{Z_{k}}(z)$, of the residual fast fading component, $Z_{\Delta}[k]$. This property does not hold for the other operators in $\mathcal{F}$. The probability in (20) can be expressed compactly as follows:

Proposition 3:

$$
p_{k}(L[k \backslash r])=\int_{\mathcal{L}[k]} \mathbf{L}_{k} \mathbf{L}_{k-1} \ldots \mathbf{L}_{k-r+1} f_{k-r+1}(s) d s,
$$

where $\mathcal{L}[k]$ is the interval associated with the symbol $L[k]$. Proposition 3 follows from the first-order AR representation of $S_{\Delta}[k]$ given in (17) and the assumption that $\left\{Z_{\Delta}[k]\right\}$ is a sequence of independent random variables, which follows from the sampling condition (7).

\section{B. Cell Assignment and Handoff Probabilities}

The key performance metrics of interest are the cell assignment and handoff probabilities. We denote by $P_{i}[k]$ and $P_{j}[k]$ the probability that the mobile is assigned at time $k$ to base stations $B S_{i}$ and $B S_{j}$, respectively. The assignment probabilities can be expressed as follows [7]:

$$
\begin{aligned}
& P_{i}[k]=p_{k}\left(I_{0} H^{k}\right)+\sum_{r=1}^{k} p_{k}\left(I H^{r-1}\right), \\
& P_{j}[k]=p_{k}\left(J_{0} H^{k}\right)+\sum_{r=1}^{k} p_{k}\left(J H^{r-1}\right) .
\end{aligned}
$$

Similarly, we denote the handoff probability at time $k$ from $B S_{i}$ to $B S_{j}$ and from $B S_{j}$ to $B S_{i}$ by $P_{i j}[k]$ and $P_{j i}[k]$, respectively. The cell handoff probabilities for $k \geq 1$ can be expressed as follows [7]:

$$
\begin{aligned}
& P_{i j}[k]=p_{k}\left(I_{0} H^{k-1} J\right)+\sum_{r=2}^{k} p_{k}\left(I H^{r-2} J\right), \\
& P_{j i}[k]=p_{k}\left(J_{0} H^{k-1} I\right)+\sum_{r=2}^{k} p_{k}\left(J H^{r-2} I\right) .
\end{aligned}
$$

To calculate the assignment and handoff probabilities efficiently, we define a sequence of functions $\left\{g_{k}(s), k \geq 1\right\}$ as follows:

$$
g_{k}(s) \triangleq \mathbf{I}_{k-1} f_{k-1}(s)+\mathbf{H}_{k-1} g_{k-1}(s), \quad k \geq 2,
$$

with the initialization $g_{1}(s)=\mathbf{I}_{0} f_{0}(s)$. The probability of assignment to $B S_{i}$ can be computed recursively as follows ${ }^{1}$.

\section{Proposition 4:}

$$
P_{i}[k]=p_{k}(I)+\int_{H} g_{k}(s) d s, \quad k \geq 1,
$$

with the initial condition $P_{i}[0]=p_{0}\left(I_{0}\right)$. The probability $p_{k}(I)$ is given as follows:

$$
p_{k}(I)=\iint_{s+z \in I} f_{k}(s) f_{Z_{k}}(z) d s d z, \quad k \geq 0 .
$$

The proof follows from Propositions 2 and 3, together with expression (23) for the assignment probability and definition (26). Proposition 4 provides an efficient procedure for computing the assignment probabilities. Note that the second term on the right side of (27) does not depend on the residual relative fast fading component. The dependence of the assignment probabilities on the residual fast fading component is completely captured by the term $p_{k}(I)$.

The handoff probability $P_{i j}[k]$ can also be expressed ${ }^{2}$ in terms of the functions $g_{k}(s)$.

\section{Proposition 5:}

$$
\begin{aligned}
P_{i j}[k] & =\iint_{s+z \in J} g_{k}(s) f_{Z_{k}}(z) d s d z, \quad k \geq 2, \\
P_{i j}[1] & =p_{1}\left(I_{0} J\right)
\end{aligned}
$$

\section{Crossover Point and Mean Number of Handoffs}

Two important handoff performance metrics that can be derived from the assignment and handoff probabilities are the crossover point and the mean number of handoffs (cf. [5], [7]). The crossover point $C$ is defined for the straightline trajectory connecting two base stations $B S_{i}$ and $B S_{j}$ as the point at which the probability of the mobile station being assigned to $B S_{i}$ drops below 0.5:

$$
C=\arg \min \left\{1 \leq k \leq K: P_{i}[k]<0.5\right\} .
$$

The mean number of handoffs, $\bar{N}_{h o}$, along a trajectory in which handoffs occur between two candidate base stations $B S_{i}$ and $B S_{j}$ is given by

$$
\bar{N}_{h o}=\sum_{k=1}^{K}\left(P_{i j}[k]+P_{j i}[k]\right) .
$$

\footnotetext{
${ }^{1}$ The result for assignment to $B S_{j}$ is analogous.

${ }^{2}$ The result for handoff from $B S_{j}$ to $B S_{i}$ is analogous.
} 

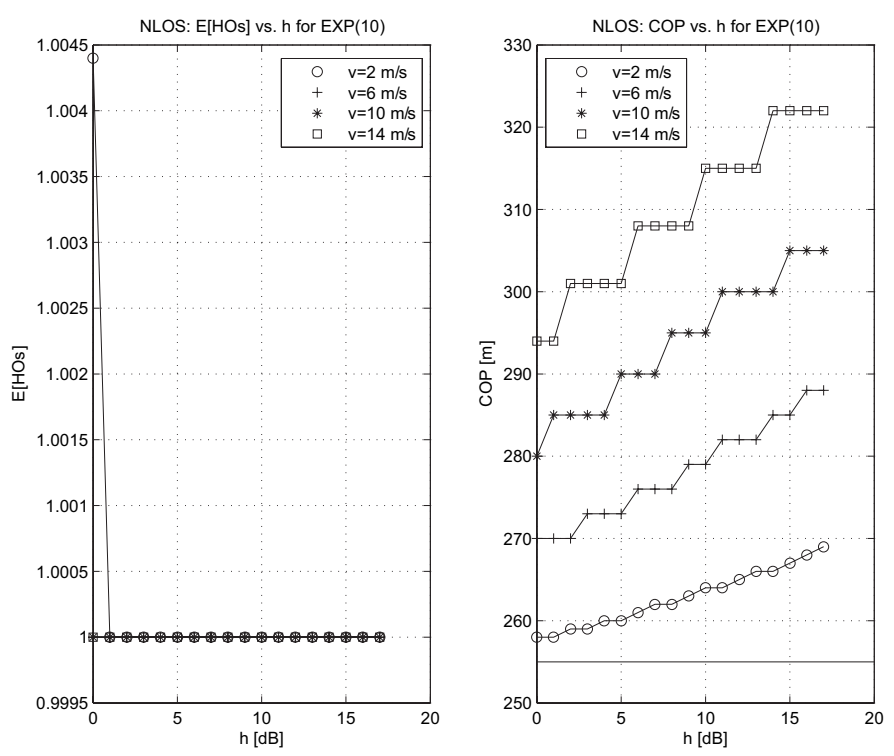

Fig. 4. Mean number of handoffs and crossover point for NLOS scenario using $\mathrm{CA}_{\exp }(10)$.
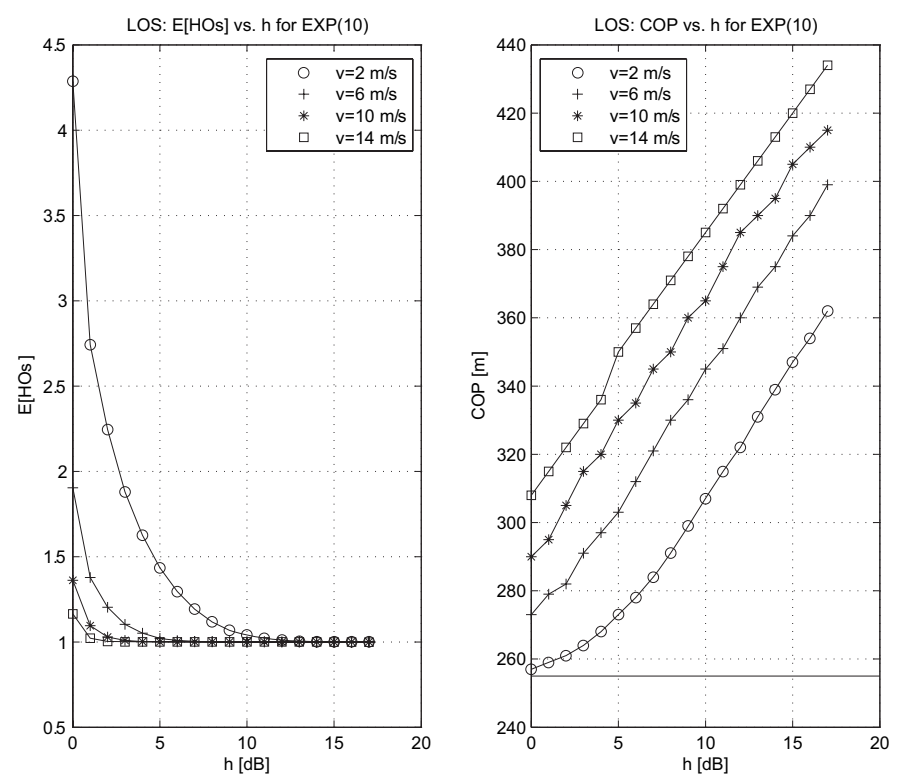

Fig. 5. Mean number of handoffs (HOs) and crossover point (COP) for LOS scenario using $\mathrm{CA}_{\exp }(10)$.

\section{Numerical Results}

In this section, we present numerical results illustrating handoff performance under local averaging for both lineof-sight (LOS) and non-line-of-sight (NLOS) trajectories, as shown in Fig. 1. For the handoff scenarios considered in our study, the path loss is assumed to follow the two-slope model discussed in Section II-A with $\mu_{i}=2, \beta_{i}=2$ and $g=150 \mathrm{~m}$ for $i=0,1,2,3$. For the NLOS trajectory, the corner effect is assumed to take place $5 \mathrm{~m}$ into the corner, so that the mobile moving from $B S_{0}$ to $B S_{1}$ experiences the corner effect at a distance of $255 \mathrm{~m}$ from $B S_{0}$. As discussed earlier, the corner effect occurs as the mobile turns around the corner of a street block, resulting in a large drop in received signal strength from the serving base station over a short distance.
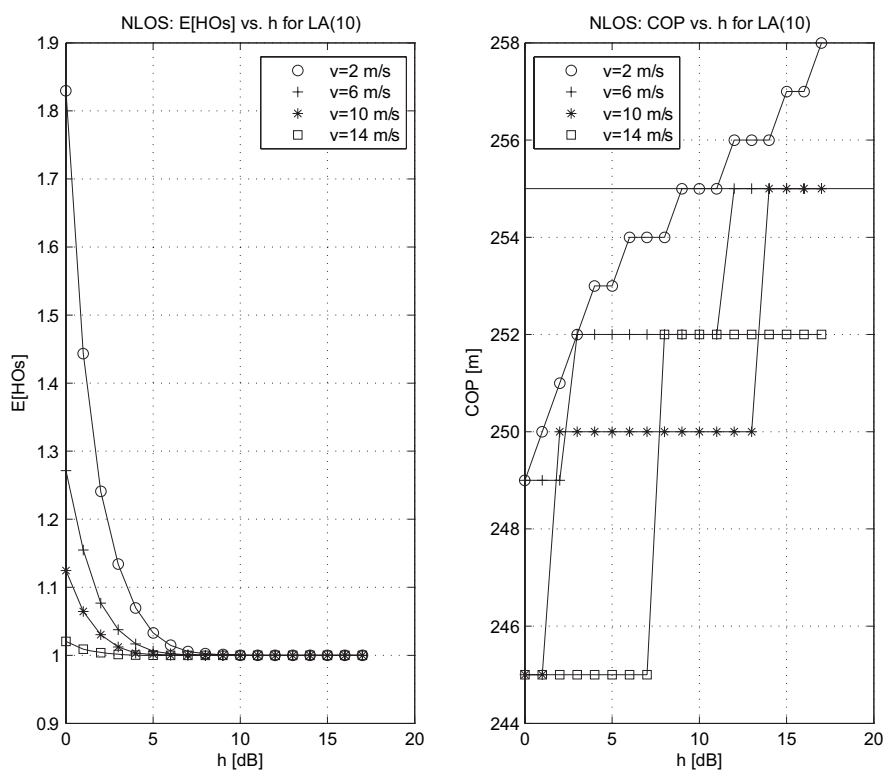

Fig. 6. Mean number of handoffs (HOs) and crossover point (COP) for NLOS scenario using $\operatorname{LA}_{\text {rect }}(10)$.

We assume correlated lognormal shadowing with a standard deviation of $\sigma_{W_{i}}=6 \mathrm{~dB}$. The hysteresis level is assumed to be independent of the base station and is denoted simply by $h$. The decay factor $d_{0}$ in (4) is set such that shadows are decorrelated to $0.1 \sigma_{W_{i}}^{2}$ at $46 \mathrm{~m}$, i.e., $d_{0}=19.98 \mathrm{~m}$. The sampling time interval for handoff decision instants is chosen as $\tau_{h}=0.48 \mathrm{~s}$, which is the same as in the GSM standard [17]. Performance curves were obtained over a range of mobile speeds, i.e., 2, 6,10 , and $14 \mathrm{~m} / \mathrm{s}$.

Figs. 4 and 5 show the handoff performance curves when conventional exponential averaging is used in the NLOS and LOS scenarios, respectively. The exponential averaging filter is given by

$$
f_{a v}[n]=\frac{e^{-n / N_{a v}}}{1-e^{-1 / N_{a v}}}, \quad n=0,1, \cdots,
$$

where $N_{a v}=10$. We denote this averaging technique by $\mathrm{CA}_{\exp }(10)$. In the NLOS scenario, the mean number of handoffs is approximately one over the entire range of hysteresis values. Here, the exponential averaging filter is able to eliminate the effect of fast fading with respect to the mean number of handoffs. The main point to observe from Figs. 4 and 5 is the large spread in the crossover point curves, indicating a high sensitivity to the mobile speed, in both the NLOS and LOS scenarios.

Figs. 6 and 7 show the handoff curves in the NLOS and LOS scenarios when local averaging with a rectangular window with $N_{a v}=10$ sample points is used. The averaging filter in this case is denoted by $\operatorname{LA}_{\text {rect }}(10)$. The mean number of handoffs under conventional averaging is smaller than when local averaging is applied, as should be expected. However, a much better tradeoff between the mean number of handoffs and the crossover point can be achieved with the local averaging technique. For example, if the hysteresis value lies in the range $5-10 \mathrm{~dB}$, the mean number of handoffs for the NLOS scenario is approximately one, while the crossover 

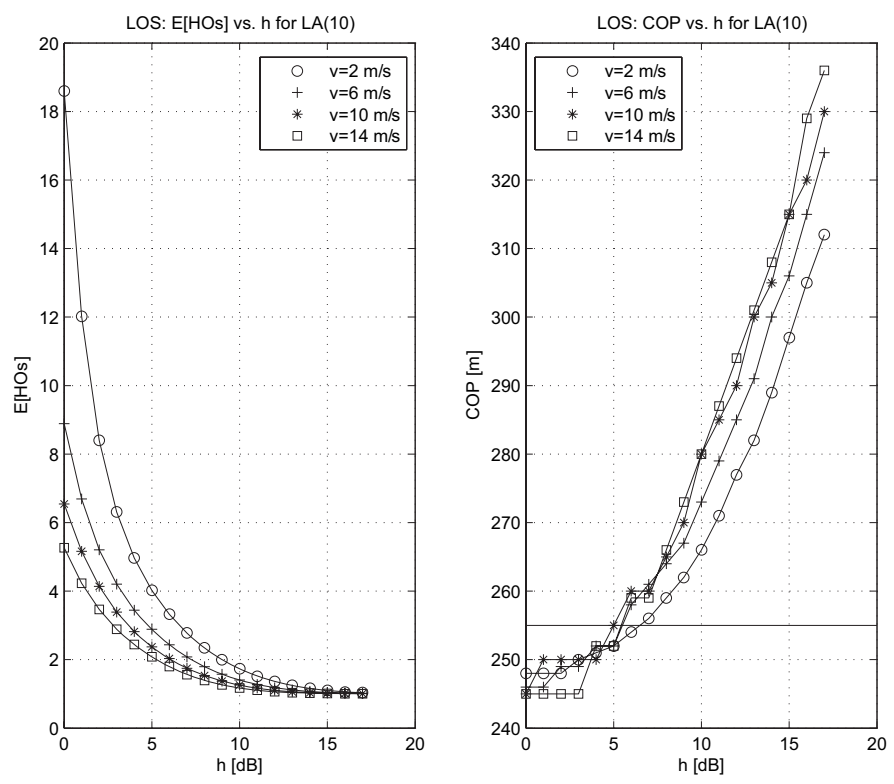

Fig. 7. Mean number of handoffs (HOs) and crossover point (COP) for LOS scenario using $\operatorname{LA}_{\text {rect }}(10)$.

point lies in the range $245-255 \mathrm{~m}$ for the entire range of mobile speeds. In the LOS case, for the same hysteresis range, the mean number of handoff lies in the range $1-4$ and the crossover point lies in the range $252-280 \mathrm{~m}$. Observe that when conventional averaging is used, there is no hysteresis value in the NLOS scenario that can be chosen to achieve a crossover point less than $260 \mathrm{~m}$. In the LOS scenario, the best crossover point performance is achieved when $h=0 \mathrm{~dB}$, in which case the crossover point lies in the range $260-310 \mathrm{~m}$, corresponding to a mean number of handoffs in the range $1-4$. We point out that the mean number of handoffs under local averaging could be further reduced using a drop timer mechanism (cf. [18]). Our results show that local averaging achieves superior handoff performance over a wide range of mobile speeds without the need for velocity estimation.

\section{CONCLUSION}

We proposed a local averaging technique for handoff algorithms to reduce handoff delay. The local averaging technique is able to track sudden changes in the received signal strength and does not require adaptation to the mobile station velocity. We presented a general framework for analyzing handoff performance with respect to the averaging method and developed an efficient numerical procedure to evaluate the performance of hysteresis-based handoff algorithms employing local averaging. Our numerical results showed that the local averaging technique significantly improves handoff performance for both line-of-sight (LOS) and non-line-ofsight (NLOS) mobile trajectories. The performance gain with respect to conventional averaging increases with the speed of the mobile. By reducing the impact of corner effects, local averaging can greatly improve handoff performance in microcellular environments.

\section{APPENDIX}

\section{A. Proof of Proposition 1}

The autocovariance function of $X_{\Delta}[k]$ can be expressed as

$$
\begin{aligned}
K_{X_{\Delta}}(l) & =K_{S_{\Delta}}(l)+K_{Z_{\Delta}}(l) \\
& =K_{\bar{W}_{\Delta}}(l q)+K_{\bar{R}_{\Delta}}(l q),
\end{aligned}
$$

where (31) follows from independence of the processes $\left\{S_{\Delta}[k]\right\}$ and $\left\{Z_{\Delta}[k]\right\}$ and (32) follows from a property of subsampling. We have that

$$
\begin{aligned}
K_{\bar{W}_{\Delta}}(m) & =f_{a v}[m] \star f_{a v}[-m] \star K_{W_{\Delta}}(m) \\
& =\alpha\left(N_{a v}, v\right) K_{W_{\Delta}}(m),
\end{aligned}
$$

where $\alpha\left(N_{a v}, v\right)$ is defined in (14). Equation (33) follows from a property of discrete-time linear filters, while (34) is derived using (16). Assuming that the sampling condition of (7) is satisfied, the samples $R_{\Delta}[k]$ can be considered independent, so that $K_{R_{\Delta}}(m)=\frac{50}{3(\ln 10)^{2}} \delta[m]$. We then have

$$
\begin{aligned}
K_{\bar{R}_{\Delta}}(m) & =f_{a v}[m] \star f_{a v}[-m] \star K_{R_{\Delta}}(m) \\
& =\beta\left(N_{a v}\right) \delta[m],
\end{aligned}
$$

where $\beta\left(N_{a v}\right)$ is defined in (15). Combining (32), (34), and (36) yields the result.

\section{REFERENCES}

[1] O. Grimlund and B. Gudmundson, "Handoff strategies in microcellular systems," in Proc. IEEE Veh. Tech. Conf. (VTC), 1991, pp. 505-510.

[2] J. M. Holtzman and A. Sampath, "Adaptive averaging methodology for handoffs in cellular systems," IEEE Trans. Vehic. Technol., vol. 44, no. 1, pp. 59-66, Feb. 1995.

[3] M. D. Austin and G. L. Stüber, "Velocity adaptive handoff algorithms for microcellular systems," IEEE Trans. Vehic. Technol., vol. 43, no. 3, pp. 549-561, Aug. 1994.

[4] C. Xiao, "Estimating velocity of mobiles in EDGE systems," in Proc. IEEE Int. Conf. Comm. (ICC), New York City, Apr./May 2002, pp. 32403244.

[5] R. Vijayan and J. M. Holtzman, "A model for analyzing handoff algorithms," IEEE Trans. Vehic. Technol., vol. 42, no. 3, pp. 351-356, Aug. 1993.

[6] N. Zhang and J. M. Holtzman, "Analysis of handoff algorithms using both absolute and relative measurements," IEEE Trans. Vehic. Technol., vol. 45, no. 1, pp. 174-179, Feb. 1996.

[7] A. E. Leu and B. L. Mark, "A discrete-time approach to analyze hard handoff performance in cellular networks," IEEE Trans. Wireless Commun., vol. 3, no. 5, pp. 1721-1733, Sept. 2004.

[8] V. V. Veeravalli and O. E. Kelly, "A locally optimal handoff algorithm for cellular communications," IEEE Trans. Veh. Technol., vol. 46, no. 3 , pp. 603-609, Aug. 1997.

[9] P. Marichamy and S. Chakrabarti, "On threshold setting and hysteresis issues of handoff algorithms," in IEEE Pers. Wireless Comm. Conf., 1999, pp. 436-440.

[10] D. Wong and D.C. Cox, "Two-state pattern recognition handoffs for corner-turning situations," IEEE Trans. Vehic. Technol., vol. 50, no. 2, pp. 354-363, March 2001.

[11] A. E. Leu and B. L. Mark, "Discrete-time level-crossing analysis of soft handoff performance in cellular networks," IEEE Trans. Inform. Theory, vol. 52, no. 7, pp. 3283-2390, July 2006.

[12] H. Suzuki, "A statistical model for urban radio propagation," IEEE Trans. Commun., vol. 25, no. 7, pp. 673-680, July 1977.

[13] P. Harley, "Short distance attenuation measurements at $900 \mathrm{MHz}$ and $1.8 \mathrm{GHz}$ using low antenna heights for microcells," IEEE J. Select. Areas Commun., vol. 7, pp. 5-11, Jan. 1989.

[14] M. Gudmundson, "Correlation model for shadowing fading in mobile radio systems," Electron. Letters, vol. 27, no. 23, pp. 2145-2146, Nov. 1991.

[15] G. L. Stüber, Principles of Mobile Communication. Kluwer Academic Press, 2nd edition, 2002. 
[16] W. Sun and X. Zhou, "Reconstruction of band-limited signals from local averages," IEEE Trans. Inform. Theory, vol. 48, no. 11, pp. 2955-2963, Nov. 2002.

[17] ETSI (European Telecommunications Standards Institute), "Digital cellular telecommunications system (Phase 2+) (GSM); Universal Mobile Telecommunications System (UMTS); Handoff procedures," ETSI TS 123 009, Dec. 2001.

[18] A. E. Leu and B. L. Mark, "An efficient timer-based hard handoff algorithm for cellular networks," in Proc. IEEE Wireless Comm. and Networking Conf. (WCNC), New Orleans, LA, Mar. 2003, vol. 2, pp. $1207-1212$.

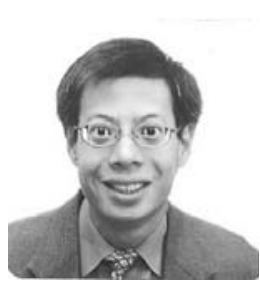

Brian L. Mark (M'91) received the B.A.Sc. degree in Computer Engineering with an option in Mathematics from the University of Waterloo, Canada, in 1991 and the Ph.D. in Electrical Engineering from Princeton University, Princeton, NJ, in 1995.

He was a Research Staff Member at the C\&C Research Laboratories, NEC USA, from 1995 to 1999. In 1999, he was on part-time leave from NEC as a visiting researcher at Ecole Nationale Supérieure des Télécommunications in Paris, France. In 2000, he joined the Dept. of Electrical and Computer Engineering at George Mason University, where he is currently an Associate Professor. His research interests lie broadly in the design, modeling, and analysis of communication systems, computer systems, and communication networks.

He was co-recipient of the best conference paper award for IEEE Infocom'97. He received a National Science Foundation CAREER Award in 2002. Currently, he is serving as an Associate Editor for IEEE Transactions on Vehicular Technology.

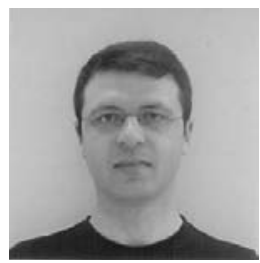

Alexe E. Leu (S'97, M'06) received the B.S. and M.S. degrees in Electronics and Telecommunications from Politechnica University of Bucharest, Romania, in 1995 and 1996, respectively and the Ph.D. degree Electrical and Computer Engineering from George Mason University, Fairfax, VA in 2003.

From 1999 to 2001, he was a Design Engineer in the R\&D Department at LCC International Inc, McLean, VA. Since 2003, he has been a Senior Research Engineer at Shared Spectrum Company, Vienna, VA. His research interests are in cognitive radio; design, modeling, and performance evaluation of wireless network architectures and protocols; integrating spatial and temporal processing for mobile networks; cross-layer design for wireless networking; and statistical modeling of wireless channels. 